\title{
Master singular behavior from correlation length measurements for seven one-component fluids near their gas-liquid critical point
}

\author{
Yves Garrabos*, Fabien Palencia, Carole Lecoutre, Can J. Erkey** \\ ESEME-CNRS, ICMCB-UPR 9048, Université Bordeaux 1, \\ 87 avenue du Docteur Albert Schweitzer, F-33608 Pessac France. and \\ **on sabbatical leave from Department of Chemical Engineering, \\ University of Connecticut, Storrs, CT 06074 USA * \\ Bernard Le Neindre \\ LIMHP-CNRS-UPR 1311, Université Paris 13, Avenue Jean Baptiste Clément, F-93430 Villetaneuse France.
}

(Dated: 27 July 2005)

\begin{abstract}
We present the master (i.e. unique) behavior of the correlation length, as a function of the thermal field along the critical isochore, asymptotically close to the gas-liquid critical point of xenon, krypton, argon, helium 3, sulfur hexafluoride, carbon dioxide and heavy water. It is remarkable that this unicity extends to the correction-to-scaling terms. The critical parameter set which contains all the needed information to reveal the master behavior, is composed of four thermodynamic coordinates of the critical point and one adjustable parameter which accounts for quantum effects in the helium 3 case. We use a scale dilatation method applied to the relevant physical variables of the onecomponent fluid subclass, in analogy with the basic hypothesis of the renormalization theory. This master behavior for the correlation length satisfies hyperscaling. We finally estimate the thermal field extent, where the critical crossover of the singular thermodynamic and correlation functions deviate from the theoretical crossover function obtained from field theory.
\end{abstract}

PACS numbers: 64.60.-i, 05.70.Jk, 64.70.Fx

\section{INTRODUCTION}

Close to the gas-liquid critical point of a onecomponent fluid, the knowledge of the correlation length $\xi$, i.e. the size of the critical fluctuations of the order parameter, is one among the most important challenge to provide better critical phenomena understanding, in particular for hyperscaling and crossover descriptions. The correlation length measurements $\xi(\Delta T)$ as a function of the temperature distance $\Delta T=T-T_{c}$ to the critical point along the critical isochore $\rho=\rho_{c}$, in the homogeneous range $T>T_{c}$, have been published for Xe [1, 2], $\mathrm{Kr}[3], \mathrm{Ar}[4],{ }^{3} \mathrm{He}$ [5, 6], $\mathrm{SF}_{6}$ [7, 8], $\mathrm{CO}_{2}$ [9, 10], and $\mathrm{D}_{2} \mathrm{O}[11,12] . T\left(T_{c}\right)$ is the temperature (critical temperature). $\rho\left(\rho_{c}\right)$ is the density (critical density). We report an analysis of these data using the scale dilatation method initially proposed by one of us [13, 14], which was recently upgraded [15] to account for quantum effects in light fluids such as ${ }^{3} \mathrm{He}$. The basic information of the scale dilatation method is given by the four coordinates which localize the critical point on the experimental $p$ (pressure), $v_{\bar{p}}$ (particle volume), $T$ (temperature), phase surface. In addition, a single well-defined adjustable parameter, noted $\Lambda_{q e}^{*}$, characterizes the quantum contribution at $T \cong T_{c}$. Considering such a minimum set of critical parameters, the two main objectives of this paper are:

i) to phenomenologically observe the master (i.e.

\footnotetext{
*Electronic address: garrabos@icmcb-bordeaux.cnrs.fr
}

unique) singular behavior of the correlation length for the universality subclass of the one-component fluids. For this we simply use the appropriate scale dilatation of $\Delta T$ and $\xi$, showing the master behavior without exact knowledge of its singular functional form;

ii) to fit the resulting master curve by a mean crossover function obtained from the recent results [16] of the massive renormalization scheme [17, 18, 19], valid for the complete universality class of the three-dimensional (3D) uniaxial symmetrical Ising like systems [20].

This paper is organized as follows. Section 2 provides the data sources. Only are considered the effective fitting results of the correlation length measurements which have been published in the litterature. After the introduction of the four critical coordinates which characterize each one-component fluid, we recall in Section 3 the essential features of the singular behavior of the correlation length. From a brief analysis based on the corresponding state scheme, we illustrate also the well-known failure of the classical theories of critical phenomena 21]. Section 4 presents the application of the scale dilatation method to the physical (field) variables of the fluid subclass, leading to the master singular behavior observed. A fitting (two-terms) power law equation, which satisfies universal features of asymptotic hyperscaling and (one-term) critical crossover valid in the preasymptotic domain [18], is also proposed in this Section 4. Then the fitting by a well-defined and complete mean crossover function obtained from the massive renormalization scheme, is made in Section 5. A brief analysis of the validity range of the classical-to-critical crossover description is given before concluding in Section 6. 


\section{THE DATA SOURCES}

The data sources are obtained from turbidity and scattering measurements as a function of $T$, which provide simultaneous determination of the susceptibility (proportional to the isothermal compressibility) and the correlation length. The measurements are performed "near the critical point", that corresponds to a finite temperature range bounded by the $\max$ and $\min$ values of $\Delta T=T-T_{c, \exp }$, where $T_{c, \exp }$ is the measured (or estimated) critical temperature in the experiments. The relative precision estimated by the authors is generally of the order of $10 \%$, but we have noted that the raw data for $\xi$, as a function of the raw data for $\Delta T$, are scarcely given in the published results to provide easy control of this uncertainty level. The authors only have systematically reported their fitting results as a function of the dimensionless temperature distance $\Delta \tau^{*}$ to the critical point, defined by

$$
\Delta \tau^{*}=\frac{\Delta T}{T_{c, \exp }}=\frac{T-T_{c, \exp }}{T_{c, \exp }}
$$

Such a normalized temperature difference is the relevant physical variable to describe the singular scaling behavior of the thermodynamic fluid properties along the critical isochore [22]. In the field theory framework [20], $\Delta \tau^{*}$ is proportional to the renormalized thermal field $t$ of the $\Phi_{d=3}^{4}(n=1)$-model for the universality class considered in the present paper (see below) which corresponds to a scalar $(n=1)$ order parameter density and a three dimensional $(d=3)$ system.

The customary functional forms used to fit the data are:

i) the effective (single term) power law divergence

$$
\xi=\xi_{0}^{+}\left(\Delta \tau^{*}\right)^{-\nu}
$$

where the free amplitude $\xi_{0}^{+}$is a fluid-dependent quantity and $\nu$ an effective critical exponent which only asymptotically $\left(\Delta \tau^{*} \rightarrow 0\right)$ takes a universal theoretical value, estimated to $\nu_{\text {Ising }} \approx 0.63$ (see [23] for updated theoretical estimations). In Eq. (2), the value of the critical exponent can be considered as an adjustable parameter when measurements are performed in a restricted temperature range at finite distance to $T_{c, \exp }$. The main characteristic of such fitting is the high correlation between the effective values of $\xi_{0}^{+}$and $\nu$, which are then highly dependent on $T_{c, \exp }$ and on the (extension and mean) values of the temperature range covered by measurements.

ii) the Wegner expansion [24], generally restricted to the following (two-term) equation,

$$
\xi=\xi_{0}^{+}\left(\Delta \tau^{*}\right)^{-\nu}\left[1+a_{\xi}^{+}\left(\Delta \tau^{*}\right)^{\Delta}\right]
$$

where $\Delta \approx 0.5$ is a universal critical exponent 23 which characterizes the leading family of the corrections to the scaling behavior. $a_{\xi}^{+}$is the fluid-dependent confluent amplitude of the first correction to scaling. In such a fitting equation, the exponents are generally fixed to their theoretical values, and only the adjustable amplitudes $\xi_{0}^{+}$ and $a_{\xi}^{+}$remain highly correlated to $T_{c, \exp }$. Moreover the contribution of the first confluent correction term to scaling mostly appears lower than - or of the same order of magnitude as - the experimental uncertainty (reflecting an effective experimental situation where $a_{\xi}^{+}(>0) \sim 1$ and $\Delta \tau^{*} \lesssim 10^{-2}$ ).

In Table I are summarized the selected fitting results [with free (or fixed) exponents and accounting (or not) for first-order Wegner term] for the seven fluids. All these fitting results are taken from litterature (see references given in the last column of Table I)

\section{ANALYSIS FROM THE CORRESPONDING STATE SCHEME}

In Figure 1a (log-log scale; color online), are reported the curves illustrating the fitted singular behavior of the $\xi(n m)$ raw data, as a function of $T-T_{c}(K)$ (from Eqs. (2) or (3) and data of Table 1). Each curve has an extension covering the experimental temperature range (while, at the Figure 1a scale, the curve thickness illustrates the $10 \%$ uncertainty on $\xi$ measurements). The dimensional quantities make each fluid behavior clearly distinguishable (at the same value of $T-T_{c}=40 \mathrm{mK}$ for example, the $\xi$ values are covering one decade: from $5 \mathrm{~nm}$ for ${ }^{3} \mathrm{He}$, up to $50 \mathrm{~nm}$ for $\mathrm{D}_{2} \mathrm{O}$ ).

Our first analysis starts from the following characteristic set [13, 14],

$$
Q_{c, a_{\bar{p}}}^{\min }=\left\{T_{c}, v_{\bar{p}, c}, p_{c}, \gamma_{c}^{\prime}=\left[\left(\frac{\partial p}{\partial T}\right)_{v_{\bar{p}, c}}\right]_{C P}\right\}
$$

made of the four critical parameters needed to localize the critical point on the $p, v_{\bar{p}}, T$ phase surface. The selected data are given in Table II. The subscript $c$ corresponds to the critical parameters, the subscript $a_{\bar{p}}$ recall the thermodynamic potential (see below) used to construct its associated phase surface, while the subscript $\bar{p}$ corresponds to a quantity normalized per particle. The subscript $C P$ means the value at the critical point. $v_{\bar{p}}=\frac{V}{N}=\frac{m_{\bar{p}}}{\rho}$, where $V$ is the total volume of the fluid container, and $N$ is the total amount of fluid particles of individual mass $m_{\bar{p}}$ [25]. Such a phase surface of equation $\Phi_{a_{\bar{p}}}^{p}\left(p, v_{\bar{p}}, T\right)=0$ uses the two conjugated experimental variables, $p$ (intensive) and $V$ (extensive), and represents the fluid equilibrium states provided by the equation of state (e.o.s.) $p\left(T, v_{\bar{p}}\right)=-\left(\frac{\partial A}{\partial V}\right)_{T, N}=-\left(\frac{\partial a_{\bar{p}}}{\partial v_{\bar{p}}}\right)_{T}$ 26]. $A\left(\Omega_{i}\right)\left[a_{\bar{p}}\left(\omega_{i}\right)=\frac{A}{N}\right]$ is the total (per particle) Helmholtz free energy of natural variables $\Omega_{i}=(T, V, N)$ $\left[\omega_{i}=\left(T, v_{\bar{p}}\right)\right] \cdot \gamma_{c}^{\prime}$ is the common limiting direction at CP, in the $p ; T$ plane, of both the critical isochore on the homogeneous (single phase) domain $\left(T>T_{c}\right)$, and the satu- 


\begin{tabular}{|c|c|c|c|c|c|c|c|}
\hline Fluid & $\begin{array}{c}\xi_{0}^{+} \\
(\AA)\end{array}$ & $\nu$ & $a_{\xi}^{+}$ & $\Delta$ & $\begin{array}{c}\Delta T_{\max } \\
(\mathrm{K})\end{array}$ & $\begin{array}{c}\Delta T_{\min } \\
(\mathrm{K})\end{array}$ & Ref. \\
\hline$X e$ & $2.0 \pm 0.25$ & $0.63 \pm 0.05$ & & & & & [1] \\
\hline$X e$ & $1.84 \pm 0.03$ & $\begin{array}{c}0.63 \\
\text { fixed }\end{array}$ & 0.55 & $\begin{array}{c}1 / 2 \\
\text { fixed }\end{array}$ & 10 & 0.0026 & [2] \\
\hline$K r$ & $1.71 \pm 0.01$ & $\begin{array}{c}0.6304 \\
\text { fixed }\end{array}$ & $0.624 \pm 0.4$ & $\begin{array}{l}0.504 \\
\text { fixed }\end{array}$ & 20 & 0.021 & [3] \\
\hline$A r$ & $1.71 \pm 0.25$ & $0.63 \pm 0.02$ & & & 3.5 & 0.04 & [4] \\
\hline$A r$ & $1.6 \pm 0.2$ & $0.64 \pm 0.02$ & & & 3.5 & 0.04 & [4] \\
\hline${ }^{3} \mathrm{He}$ & $4.8 \pm 2.0$ & $0.59 \pm 0.04$ & & & 0.00014 & 0.000014 & [5] \\
\hline${ }^{3} \mathrm{He}$ & $2.71 \pm 0.015$ & $0.629 \pm 0.002$ & $0.732 \pm 0.007$ & $0.502 \pm 0.01$ & 0.1 & 0.000014 & [6] \\
\hline$S F_{6}$ & $1.5 \pm 0.23$ & $0.67 \pm 0.07$ & & & 0.45 & 0.038 & [7] \\
\hline$S F_{6}$ & $2.016 \pm 0.2$ & $0.6214 \pm 0.01$ & & & 1.0 & 0.048 & [8] \\
\hline $\mathrm{CO}_{2}$ & $1.94 \pm 0.2$ & $0.60 \pm 0.02$ & & & 10 & 0.01 & [9] \\
\hline $\mathrm{CO}_{2}$ & $1.50 \pm 0.09$ & $0.633 \pm 0.01$ & & & 10 & 0.023 & {$[10]$} \\
\hline $\mathrm{D}_{2} \mathrm{O}$ & $1.30 \pm 0.23$ & $0.623 \pm 0.03$ & & & 13 & 0.15 & [11] \\
\hline $\mathrm{D}_{2} \mathrm{O}$ & $1.372 \pm 0.01$ & $\begin{array}{c}0.6304 \\
\text { fixed }\end{array}$ & $0.676 \pm 0.2$ & $\begin{array}{l}0.504 \\
\text { fixed }\end{array}$ & 22 & 1.6 & {$[12]$} \\
\hline
\end{tabular}

Table I: Published values of $\xi_{0}^{+}, \nu, a_{\xi}^{+}$, and $\Delta$, obtained from fitting [with the Eqs. [23)] the turbidity and scattering measurements, in the temperature range $\Delta T_{\min } \leqslant T-T_{c} \leqslant \Delta T_{\max }$, along the critical isochore of seven one-component fluids (for data sources and the selected fitting results see the references given in the last column).

ration pressure curve - [the projection of the vapor-liquid equilibrium state] - on the heterogeneous (two phase) domain $\left(T<T_{c}\right)$. We note that the $T_{c}$ values given in Table II, which result from complete thermodynamic analysis of the phase surface, are mostly different from $T_{c, \text { exp }}$, as the $\rho_{c}=\frac{m_{\bar{p}}}{v_{\bar{p}, c}}$ values from Table II generally differ from the measured (or estimated) critical density in the selected experiments.

In a first step, from $Q_{c, a_{\bar{p}}}^{\min }$, we are able to derive dimensionless thermodynamic and correlation functions, using the scale factors,

$$
\left(\beta_{c}\right)^{-1}=k_{B} T_{c}
$$

as an energy unit, and

$$
\alpha_{c}=\left(\frac{k_{B} T_{c}}{p_{c}}\right)^{\frac{1}{d}}
$$

as a length unit. In regards to the noticeable differences in the $\xi$ values observed in Figure 1a, at a same $T-T_{c}$ value, we note the small differences in $\alpha_{c}$ values, given in the column 8 of Table II. We also note that $\alpha_{c}$, obtained only using intensive variables, is not dependent of the size $L \sim(V)^{\frac{1}{d}}$ of the container $\left(k_{B}\right.$ is the Boltzmann constant; $d=3$ ). $\alpha_{c}$ has a clear physical meaning as a length unit [13]: it represents the spatial extent of the short-ranged (Lennard-Jones like) molecular interaction [27], which allows us to define $v_{c, I}=\frac{k_{B} T_{c}}{p_{c}}$ as the volume of the microscopic critical interaction cell of each fluid.

The introduction of the two characteristic dimension- less numbers,

$$
Z_{c}=\frac{p_{c} v_{\bar{p}, c}}{k_{B} T_{c}}
$$

and

$$
Y_{c}=\gamma_{c}^{\prime} \frac{T_{c}}{p_{c}}-1
$$

allows us to rewrite the minimal set [Eq. (4)] in the more convenient form

$$
Q_{c, a_{\bar{p}}}^{\min }=\left\{\left(\beta_{c}\right)^{-1}, \alpha_{c}, Z_{c}, Y_{c}\right\}
$$

which involves one energy scale factor, one length scale factor, and two dimensionless scale factors characterizing two preferred directions to cross the critical point along the critical isotherm and the critical isochore, respectively. $Z_{c}$ is the usual critical compression factor. In addition, $\left(Z_{c}\right)^{-1}=n_{c} v_{c, I}$ is the number of particles that fill $v_{c, I}$, and the minimal set given by the equation (9) appears related to the critical interaction cell properties.

We recall that the critical compression factor $Z_{c}$, and the critical Riedel factor $\alpha_{R, c}$ - related to $Y_{c}$ by $\alpha_{R, c}=$ $Y_{c}+1-$, are also two basic tools for developing an e.o.s. for engineering fluid modeling.

Figure 1b (log-log scale; color online), gives the singular behavior of the dimensionless correlation length $\xi^{*}=\frac{\xi}{\alpha_{c}}$ as a function of the dimensionless temperature distance $\Delta \tau^{*}$ of Eq. (1), precisely obtained from the classical theory of corresponding states (here with two characteristic parameters), using $\left(\beta_{c}\right)^{-1}$ and $\alpha_{c}$ units. 

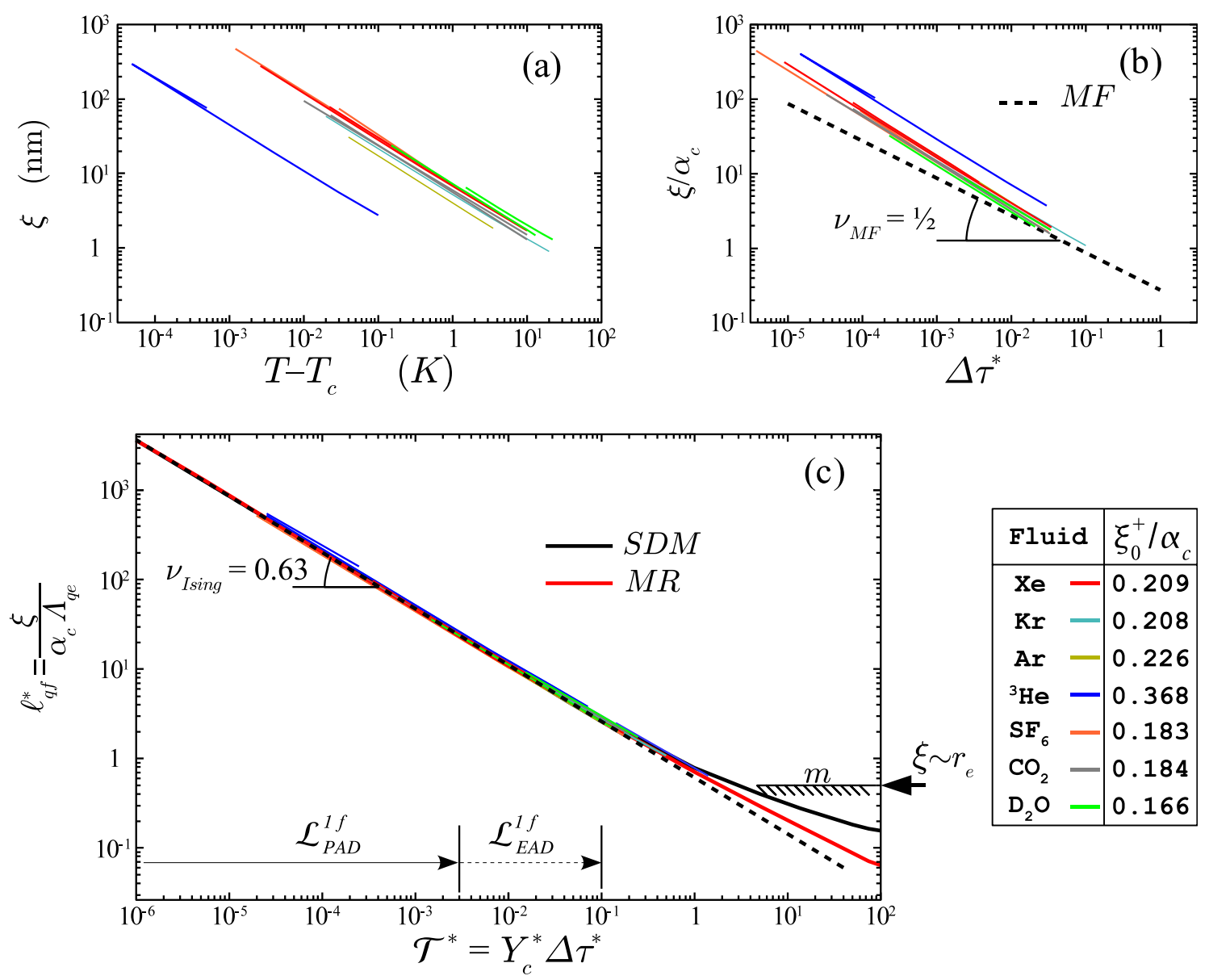

Figure 1: (Color online) a) Log-Log scale of $\xi$ as a function of $T-T_{c}>0$, along the critical isochore for Xe, $\mathrm{Kr}, \mathrm{Ar},{ }^{3} \mathrm{He}$, $\mathrm{SF}_{6}, \mathrm{CO}_{2}$, and $\mathrm{D}_{2} \mathrm{O}$. Each corresponding curve corresponds to the fit results reported in Table I, using Eqs. (2) and (3) for fitting data on the corresponding experimental range; b) Log-Log scale of the respective dimensionless variables expressed in $\alpha_{c}$ length unit and $\left(\beta_{c}\right)^{-1}$ energy unit, illustrating the failure of the classical corresponding state scheme. The expected mean field behavior with classical index $\nu_{M F}=\frac{1}{2}$ is given by the curve labelled $M F$ [see text and Eq. (10)]; c) Matched master behavior (Log-Log scale) of the renormalized correlation length $\ell_{q f}^{*}$, as a function of the renormalized thermal field $\mathcal{T}^{*}$, [see Eqs. (20) and (14), respectively]. The curves labelled $S D M$ and $M R$ correspond to the Eqs. (21) and (25), respectively. The dashed line illustrates the universal critical index $\nu_{\text {Ising }} \simeq 0.63$ for the asymptotic pure power law behavior of the uniaxial $3 \mathrm{D}$ Ising-like universality class. At large values of the renormalized thermal field $\left(\mathcal{T}^{*} \geq 3\right)$, we have illustrated by the curve $m$ a rough estimate $\ell_{q f}^{*} \approx \frac{1}{2}$ of the microscopic limit where the correlation length $\xi$ will reach the order of magnitude of the two particle equilibrium position $r_{e}$ (with $r_{e} \gtrsim \sigma$, where $\sigma$ is the size of the particle); see text. In the superimposed Table are shown color indexation for each fluid (left column) and calculated $\frac{\xi_{0}^{+}}{\alpha_{c}}$ values (right column), using Eq. (28) and Table II data.

\begin{tabular}{|c|c|c|c|c|c|c|c|c|c|c|}
\cline { 2 - 10 } Fluid & $\begin{array}{c}m_{\bar{p}} \\
\left(10^{-26} \mathrm{~kg}\right)\end{array}$ & $\Lambda_{q e}^{*}$ & $\begin{array}{c}T_{c} \\
(\mathrm{~K})\end{array}$ & $\begin{array}{c}v_{\bar{p}, c} \\
\left(\mathrm{~nm}^{3}\right)\end{array}$ & $\begin{array}{c}p_{c} \\
(\mathrm{MPa})\end{array}$ & $\begin{array}{c}\gamma_{c}^{\prime} \\
\left(\mathrm{MPa} \mathrm{K}^{-1}\right)\end{array}$ & $\begin{array}{c}\left(\beta_{c}\right)^{-1} \\
\left(10^{-21} \mathrm{~J}\right)\end{array}$ & $\begin{array}{c}\alpha_{c} \\
(\mathrm{~nm})\end{array}$ & $Z_{c}$ & $Y_{c}$ \\
\hline $\mathrm{Xe}$ & 21.803 & 1 & 289.74 & 0.19589 & 5.84 & 0.118 & 4.0003 & 0.881508 & 0.28601 & 4.85434 \\
\hline $\mathrm{Kr}$ & 13.9154 & 1 & 209.286 & 0.15292 & 5.5 & 0.1562 & 2.88951 & 0.806901 & 0.291065 & 4.94372 \\
\hline $\mathrm{Ar}$ & 6.6336 & 1 & 150.725 & 0.12388 & 4.865 & 0.172 & 2.08099 & 0.753463 & 0.2896 & 4.32882 \\
\hline${ }^{3} \mathrm{He}$ & 0.4983 & 1.11966 & 3.31555 & 0.12022 & 0.114724 & 0.11759 & 0.0457761 & 0.736198 & 0.301284 & 2.39837 \\
\hline $\mathrm{SF}_{6}$ & 24.252 & 1 & 318.70 & 0.32684 & 3.76 & 0.0835 & 4.4 & 1.054 & 0.281 & 6.08 \\
\hline $\mathrm{CO}_{2}$ & 7.308 & 1 & 304.14 & 0.15622 & 7.3753 & 0.170 & 4.2 & 0.829 & 0.274 & 6.01 \\
\hline $\mathrm{D}_{2} \mathrm{O}$ & 3.329 & 1 & 643.89 & 0.09346 & 21.671 & 0.2717 & 8.88987 & 0.743029 & 0.227829 & 7.07277 \\
\hline
\end{tabular}

Table II: Minimal set of critical parameters [see Eqs. (4) and (9)] for the seven one-component fluids of particle mass $m_{\bar{p}}$. $\Lambda_{q e}^{*} \geq 1$ [Eq. (17)] differs from unity by a nonuniversal adjustable quantity proper to the nature of the ${ }^{3} H e$ quantum particle [see text and Eqs (18) and (19)] 
Evaluation from standard mono-atomic Xe, shows the failure of the classical theory, increasing quantum effects in ${ }^{3} \mathrm{He}$, and decreasing non-spherical interaction effects in $\mathrm{D}_{2} \mathrm{O}$. The dimensionless correlation length is then covering a relative variation by a factor more than 2 at the same reduced temperature distance to the critical point (see also our calculated values of $\frac{\xi_{0}^{+}}{\alpha_{c}}$ in the inserted table on Figure 1). Moreover an illustration of the inaptness of the "mean field" exponent $\nu_{M F}=\frac{1}{2}$ to describe the classical fluctuation behavior [28] expected from Van der Waals-like theories, is shown by the curve labelled $M F$, of equation

$$
\frac{\xi_{M F}}{\alpha_{c}}=\frac{1}{4}\left(\frac{3 \sqrt{2}}{\pi}\right)^{\frac{1}{d}}\left(\Delta \tau^{*}\right)^{-\nu_{M F}}
$$

We note also that the classical approach along the critical isochore in the homogeneous domain is not able to reproduce experimental behavior at large distance to the critical point. Such a failure can be related to the number of interacting particles (here given by $\frac{1}{Z_{c}} \sim 3-4$ ), which seems too small to validate a "mean field approximation" of the attractive molecular interaction at distance greater than $\alpha_{c}$ for a fluid at critical density, as we will discuss below (see Section 5).

\section{ANALYSIS USING THE SCALE DILATATION METHOD}

As demonstrated in references 14, 29], $Z_{c}$ and $Y_{c}$ can be used as the two scale factors to formulate the dimensionless master asymptotic behavior of the one component fluid subclass. As a matter of fact, asymptotic master singular behaviors of dimensionless potentials and dimensionless correlation functions only occur [13] using appropriate dilatations of the following physical variables,

$$
\Delta \tau^{*}=k_{B} \beta_{c}\left(T-T_{c}\right)
$$

(to generate the renormalized thermal field),

$$
\Delta h^{*}=\beta_{c}\left(\mu_{\bar{p}}-\mu_{\bar{p}, c}\right)
$$

(to generate the renormalized ordering field), and

$$
\Delta m^{*}=\left(\alpha_{c}\right)^{d}\left(n-n_{c}\right)
$$

(to generate density of the renormalized order parameter - conjugated to the renormalized ordering field). These scale dilatations of the fluid variables are now defined [15] in complete formal analogy to the field theory framework [20]. Such a theoretical approach provides a comprehensive understanding of the diverging universal character of the spontaneous fluctuations of extensive variables, using the renormalization group (RG) techniques 30, 31, 32], to deal with the contributions of critical fluctuations. We briefly recall that this theoretical approach accounts for infinite degrees of freedom near the non-Gausssian (Wilson-Fisher) fixed point, throughout the Landau-Ginzburg-Wilson Hamiltonian of the $\Phi_{d=3}^{4}(n=1)$-model for the universality class of the 3D uniaxial symmetrical Ising like systems, with associated coupling constant $u_{4}>0$. In this model, the relevant pair of renormalized fields are the weakly fluctuating thermal field $t$, and the ordering field $h$ which exhibits stronger fluctuations, with $\{t=0, h=0\}$ at the isolated non-Gaussian fixed point. A single parameter $\kappa$, such that $\kappa<<\Lambda_{0}$, measures the distance to the non-Gaussian fixed point in such a way that this fixed point corresponds to $\kappa^{*}=\frac{\kappa}{\Lambda_{0}}=0 . \Lambda_{0}$ is the actual microscopic wave number, characterizing a discrete structure of matter with spacing $\left(\Lambda_{0}\right)^{-1} \cdot \kappa$ is precisely related to the inverse correlation length $\xi^{-1}$ of the fluctuations of the order-parameter $m$, conjugated to $h$, with $\ell_{q f}^{*}=\Lambda_{0} \xi=\left(\kappa^{*}\right)^{-1}$. Here $\ell_{q f}^{*}$ corresponds to the dimensionless form of the actual fluid correlation length expressed in units of $\alpha_{c}$ [Eq. [6)], also including quantum fluids (labelled $q f$ ) throughout the introduction of a dimensionless adjustable parameter $\Lambda_{q e}^{*}$ which account for the quantum effects [see below the Eq. (20) and the related discussion]. At the fixed point, the fluctuations are infinite, i.e. $\xi(t=0, h=0) \sim \infty$. Close to the nonGaussian fixed point within the critical asymptotic domain, i. e. for small values of the renormalized fields $t$ and $h$ which ensure that $\kappa<<\Lambda_{0}$, the Wegner expansions 24] represent the singular behavior of thermodynamics and correlations functions of any physical system belonging to the universality class of this $\Phi_{d=3}^{4}(n=1)$ model. In particular, the Wegner expansion of Equation (3) can be used for $\xi$. In that critical asymptotic domain, $\xi$, although finite, is still larger than $\left(\Lambda_{0}\right)^{-1}$. So that the close critical vicinity of the non-Gaussian fixed point can be defined by $\ell_{q f}^{*}=\Lambda_{0} \xi>>1$.

The renormalization introduces the two-scale universality of the physical system through analytical proportionality between the physical variables and the renormalized fields $t$ and $h$, respectively [30, 31]. In a similar manner, the scale dilatation method is defined by the following renormalization of $\Delta \tau^{*}$ and $\Delta h^{*}$ into $\mathcal{T}_{q f}^{*}$ and $\mathcal{H}_{q f}^{*}$

$$
\begin{gathered}
\mathcal{T}_{q f}^{*} \equiv \mathcal{T}^{*}=Y_{c} \Delta \tau^{*} \\
\mathcal{H}_{q f}^{*}=\left(\Lambda_{q e}^{*}\right)^{2} \mathcal{H}^{*}=\left(\Lambda_{q e}^{*}\right)^{2}\left(Z_{c}\right)^{-\frac{d}{2}} \Delta h^{*}
\end{gathered}
$$

respectively. Correspondingly, the renormalization of the order parameter $\Delta m^{*}$ into $\mathcal{M}_{q f}^{*}$ reads as follows

$$
\mathcal{M}_{q f}^{*}=\Lambda_{q e}^{*} \mathcal{M}^{*}=\Lambda_{q e}^{*}\left(Z_{c}\right)^{\frac{d}{2}} \Delta m^{*}
$$

Obviously, $\mathcal{T}^{*}, \mathcal{H}^{*}$ and $\mathcal{M}^{*}$, are the renormalized variables defined for non-quantum fluids, for which the 
nonuniversal wave number reads $\Lambda_{0}=\frac{1}{\alpha_{c}}$, since $\alpha_{c}$ is the single explicit length unit. In Eq. (14), the identity $\mathcal{T}_{q f}^{*} \equiv \mathcal{T}^{*}$ means that the quantum effects are only accounted for at $T \cong T_{c}$. In Eqs. (15) and (16), the dimensionless parameter

$$
\Lambda_{q e}^{*}=1+\lambda_{c}
$$

accounts for quantum effects on the microscopic wave number $\Lambda_{0}$ at $T \cong T_{c}$ [15], in such a relative phenomenological way that

$$
\Lambda_{0} \Lambda_{q e}^{*}=\frac{1}{\alpha_{c}}
$$

with

$$
\lambda_{c}=\lambda_{q, f} \frac{\Lambda_{T, c}}{\alpha_{c}}
$$

$\lambda_{q, f}$ (with $\lambda_{q, f}>0$ ), is thus a nonuniversal adjustable number which accounts for statistical contribution due to the nature (boson, fermion, etc.) of the quantum particle. $\Lambda_{T, c}=\frac{h_{P}}{\left(2 \pi m_{\bar{p}} k_{B} T_{c}\right)^{\frac{1}{2}}}$ is the de Broglie thermal wavelength at $T=T_{c}, h_{P}$ is the Planck constant (the subscript $P$ is here added to make a distinction with the field theory ordering field $h$ ). The Eq. (18) preserves the same length scale unit for thermodynamic and correlations functions.

Therefore, the renormalized dimensionless correlation length is given by

$$
\ell_{q f}^{*}=\left(\kappa^{*}\right)^{-1}=\Lambda_{0} \xi=\frac{\xi^{*}}{\Lambda_{q e}^{*}}=\frac{\xi}{\alpha_{c} \Lambda_{q e}^{*}}
$$

and the corresponding dilatation of the dimensionless axis $\Delta \tau^{*}$ and $\frac{\xi}{\alpha_{c}}$ into $\mathcal{T}^{*}$ and $\ell_{q f}^{*}$ are then defined by Eqs. (14) and (20), respectively, only using $Q_{c, a_{\bar{p}}}^{\min }$ and $\Lambda_{q e}^{*}$.

The expected collapsing onto the master behavior obtained from application of this scale dilatation method to the physical variables is shown in Figure 1c (log-log scale; color online), independently of any theoretical form used to represent this master behavior. The scatter between the curves now corresponds to their estimated precision (10\%) for each fluid correlation length.

Since the scale dilatation of the physical variables [see equations (14) and (15)] is analogous to the basic hypotheses of the renormalization group, we expect that the master asymptotic singularities present the universal features of the universality class. Specially within the preasymptotic domain, the observed divergence of $\ell_{q f}^{*}$ can be represented by the following two-term Wegner expansion

$$
\ell_{q f}^{*}=\mathcal{Z}_{\xi}^{+}\left(\mathcal{T}^{*}\right)^{-\nu}\left[1+\mathcal{Z}_{\xi}^{1,+}\left(\mathcal{T}^{*}\right)^{\Delta}\right]
$$

where the leading amplitude $\mathcal{Z}_{\xi}^{+}=0.57$ and the first confluent amplitude $\mathcal{Z}_{\xi}^{1,+}=0.377$ have master (i.e. constant) values for the pure fluid subclass (see the Refs.

\begin{tabular}{|c|c|c|c|c|c|c|}
\hline & exponent & $\mathbb{Z}_{\xi}^{+}$ & $S_{2}$ & $i$ & $X_{\xi, i}$ & $Y_{\xi, i}$ \\
\hline$\nu$ & 0.6303875 & 2.121008 & 22.9007 & 1 & 40.0606 & -0.098968 \\
\hline$\Delta$ & 0.50189 & & & 2 & 11.9321 & -0.15391 \\
\hline$\Delta_{M F}$ & 0.5 & & & 3 & 1.90235 & -0.00789505 \\
\hline & & & & & $\mathbb{Z}_{\xi}^{1,+}$ & 5.81623 \\
\hline
\end{tabular}

Table III: Values of the universal exponents and constant parameters of Eqs. (22), (23) and (30).

14, 29] for details making reference to critical xenon behavior 33 in order to obtain these master values). This result, obtained from master singular behavior of thermodynamic properties satisfies asymptotic hyperscaling and extends the scaling assertions first proposed by Widom 22. for the equation of state of the one-component fluid.

\section{MEAN CROSSOVER FUNCTION FROM THE MASSIVE RENORMALIZATION SCHEME}

We can now consider the "min" and "max" accurate expressions of the complete classical-to-critical crossover recently proposed by Bagnuls and Bervillier [16]. From the numeric values of the parameters of the generic functions $F_{\min }(t)$ and $F_{\max }(t)$ given in Tables I and II of reference [16], we have derived the numerical values of parameters associated to the mean crossover functions which reproduce as closely as possible the error treatment initially made by the authors from their generic functions. Such a mean crossover function for the inverse correlation length reads as follows

$$
\left[\ell^{*}(t)\right]^{-1}=\mathbb{Z}_{\xi}^{+}(t)^{\nu} \prod_{i=1}^{i=3}\left[1+X_{\xi, i} t^{D(t)}\right]^{Y_{\xi, i}}
$$

with

$$
D(t)=\frac{\Delta+\Delta_{M F} S_{2} \sqrt{t}}{1+S_{2} \sqrt{t}}
$$

and

$$
t=\vartheta\left|\Delta \tau^{*}\right|
$$

All the critical exponents $\left(\nu, \Delta, \Delta_{M F}\right)$ and the constants $\left(\mathbb{Z}_{\xi}^{+}, X_{\xi, i}, Y_{\xi, i}, S_{2}\right)$ are given in Table III. The adjustable parameter $\vartheta$ introduces the non-universality proper to each selected system.

To fit each curve of Figure 1a with equations (22) to (24), we introduce one asymptotic (system-dependent) prefactor $\lambda_{0}$, of dimension [length $]^{-1}$, from the following equation

$$
\frac{1}{\xi\left(\Delta \tau^{*}\right)}=\lambda_{0} \mathbb{Z}_{\xi}^{+}\left(\Delta \tau^{*}\right)^{\nu} \prod_{i=1}^{i=3}\left[1+X_{\xi, i} t^{D(t)}\right]^{Y_{\xi, i}}
$$

where we note that the leading term is now a unique function of $\Delta \tau^{*}$, as proposed by Bagnuls and Bervillier 
[16]. In such a fitting procedure, $\vartheta$ is readily seen as a crossover parameter associated with one irrelevant physical field. The prefactor $\lambda_{0}$ satisfies to the two-scale universal feature of this universality class, associated with the two relevant physical fields (only two among all these prefactors are characteristics of the non-universality of the selected system). From our definition of the length unit, we can rewrite $\lambda_{0}$ as follows

$$
\lambda_{0}=\frac{1}{\alpha_{c}} \ell_{0}^{*}
$$

leading to the following modification of the above equation (25)

$$
\frac{\alpha_{c}}{\xi\left(\Delta \tau^{*}\right)}=\ell_{0}^{*} \mathbb{Z}_{\xi}^{+}\left(\Delta \tau^{*}\right)^{\nu} \prod_{i=1}^{i=3}\left[1+X_{\xi, i} t^{D\left(t^{*}\right)}\right]^{Y_{\xi, i}}
$$

Now it is easy to understand that the restricted analysis of the two-term Wegner expansion provides complete materials for the unequivocal determination of the two adjustable parameters $\ell_{0}^{*}$ and $\vartheta$. For example, the asymptotic term to term comparison of equation (3) and inverse equation (27), provides the following two relations

$$
\xi_{0}^{+}=\alpha_{c}\left(\ell_{0}^{*} \mathbb{Z}_{\xi}^{+}\right)^{-1}
$$

and

$$
a_{\xi}^{+}=\mathbb{Z}_{\xi}^{1,+}(\vartheta)^{\Delta}
$$

where

$$
\mathbb{Z}_{\xi}^{1,+}=-\sum_{i=1}^{i=3} X_{\xi, i} Y_{\xi, i}
$$

The value of the constant amplitude $\mathbb{Z}_{\xi}^{1,+}$ is given in Table III. However, we note that the quantum correction parameter disappears in such a standard estimation of the leading prefactor (and leading amplitude) when the two scale factors are unknown [33].

$>$ From the scale dilatation method, to fit the master curve of Figure 1c with equations (22) to (24), need to introduce two master (i.e. constant) parameters, $\ell_{0}^{\{1 f\}}$ and $\vartheta^{\{1 f\}}$, that are characteristics of the one-component fluid subclass, using the crossover modeling equation

$$
\begin{aligned}
\frac{1}{\ell_{q f}^{*}\left(\mathcal{T}^{*}\right)}= & \ell_{0}^{\{1 f\}} \mathbb{Z}_{\xi}^{+}\left(\vartheta^{\{1 f\}} \mathcal{T}^{*}\right)^{\nu} \\
& \times \prod_{i=1}^{i=3}\left[1+X_{\xi, i}\left(\vartheta^{\{1 f\}} \mathcal{T}^{*}\right)^{D\left(\vartheta^{\{1 f\}} \mathcal{T}^{*}\right)}\right]^{Y_{\xi, i}}
\end{aligned}
$$

In equation (31),

$$
\ell_{0}^{\{1 f\}}=\left[\mathcal{Z}_{\xi}^{+} \mathbb{Z}_{\xi}^{+}\left(\vartheta^{\{1 f\}}\right)^{\nu}\right]^{-1}
$$

and

$$
\vartheta^{\{1 f\}}=\left(\frac{\mathcal{Z}_{\xi}^{1,+}}{\mathbb{Z}_{\xi}^{1,+}}\right)^{\frac{1}{\Delta}}
$$

in order to agree with the two-term asymptotic behavior given by equation (21). The mandatory relation between the relevant field $t$ of the $\Phi_{d=3}^{4}(1)$-model and the master field $\mathcal{T}^{*}$ of the fluid subclass reads as follows

$$
t=\vartheta^{\{1 f\}} \mathcal{T}^{*}
$$

From $\mathbb{Z}_{\xi}^{+}, \mathbb{Z}_{\xi}^{1,+}, \mathcal{Z}_{\xi}^{+}$, and $\mathcal{Z}_{\xi}^{1,+}$ values we obtain $\vartheta^{\{1 f\}}=$ 0.004288 and $\ell_{0}^{\{1 f\}}=25.699$. We note that the master prefactor $\ell_{0}^{\{1 f\}}$ is attached to the correlation length behaviors above and below the critical temperature, while the master crossover parameter $\vartheta\{1 f\}$ is the same for any property along the critical isochore, above and below the critical temperature. The respective curves labelled $S D M$ [of equation (21)] and $M R$ [of inverse equation (31)], are illustrated in Figure 1c, with noticeable asymptotic agreement with master experimental behavior of the seven one-component fluids. The preasymptotic domain (labelled $P A D$ ), described by a Wegner expansion restricted to the first confluent correction [see equation (21)], extends up to $\mathcal{L}_{P A D}^{1 f} \lesssim 310^{-3}$ (see the corresponding arrow in $\mathcal{T}^{*}$ axis). In the extended asymptotic domain (labelled $E A D$ ) which extends up to $\mathcal{L}_{E A D}^{1 f} \lesssim 0.1$ (see the corresponding arrow in $\mathcal{T}^{*}$ axis), the observed master behavior is well-represented by the theoretical critical-to-classical crossover [see equation (31)]. From the comparison of Figures $1 \mathrm{~b}$ and 1c, we can also note that the applicability of the two-scale master behavior obtained from the scale dilatation method goes far beyond the applicability of the corresponding state method based on classical theory.

By reversing the scale dilatation method for any onecomponent fluid where $Q_{c, a_{\bar{p}}}^{\min }$ and $\Lambda_{q e}^{*}$ are known, it is easy to determine its attached two characteristic parameters $\ell_{0}^{*}$ and $\vartheta$, and to derive Eq. (3) from Eq. (21), using the following relations

$$
\begin{gathered}
\ell_{0}^{*}=\frac{1}{\Lambda_{q e}^{*}} \ell_{0}^{\{1 f\}} \\
\vartheta=Y_{c} \vartheta^{\{1 f\}} \\
\xi_{0}^{+}=\alpha_{c} \Lambda_{q e}^{*}\left(Y_{c}\right)^{-\nu} \mathcal{Z}_{\xi}^{+} \\
a_{\xi}^{+}=\mathcal{Z}_{\xi}^{1,+}\left(Y_{c}\right)^{\Delta}
\end{gathered}
$$

Equation (3) can now be readily used without any adjustable parameter (except $\Lambda_{q e}^{*}$ ). 
Since the critical behaviour predicted by RG theory asymptotically agrees with the master behavior for the one-component fluid subclass, the only remaining problem is the determination of the thermal field distance at which significant deviation between the $\Phi_{d=3}^{4}(n=1)$ model and the fluid subclass appears. To point out that such a thermal distance (noted $\mathcal{T}_{C O}^{*}$ in the following) exists, we consider the variation of the effective exponent, $\nu_{\text {eff }}=-\frac{d \ln \left(\ell_{q f}^{*}\right)}{d \ln \mathcal{T}}$, as a function of $\mathcal{T}^{*}$. In addition, it is also possible to consider the variation of the effective exponent, $\gamma_{e f f}=-\frac{d \ln \left(\mathcal{X}_{q f}^{*}\right)}{d \ln \mathcal{T}}$, which is now entirely known 34], thanks to universal features predicted by RG theory and definitions [see equations (14), (15), and (16)] of the scale dilatation. $\mathcal{X}_{q f}^{*}(\mathcal{T})=$ $\left(\frac{\partial \mathcal{M}_{q f}^{*}}{\partial \mathcal{H}_{q f}^{*}}\right)_{\mathcal{T}}=\left(\Lambda_{q e}^{*}\right)^{2-d}\left(Z_{c}\right)^{d-2} \kappa_{T}^{*}$ is the renormalized susceptibility [15], with $\kappa_{T}^{*}=p_{c} \kappa_{T} . \kappa_{T}=\frac{1}{\rho}\left(\frac{\partial \rho}{\partial T}\right)_{T}$ is the (physical) isothermal compressibility. The two limits for the $\gamma_{e f f}$-variation calculated from the $\Phi_{d=3}^{4}(1)$ model are $\gamma_{M F}=1$ (close to the Gaussian fixed point) and $\gamma_{\text {Ising }} \cong 1.24$ [23] (close to the Wilson-Fisher fixed point).

The complete results are shown in Figure 2 (color online) as a function of the renormalized thermal field $\mathcal{T}^{*}$ (lower axis), or as a function of the renormalized correlation length $\ell_{q f}^{*}$ (upper axis). We recall that $\ell_{q f}^{*}$ gives a best estimate of the ratio between the effective size $(\xi)$ of the critical fluctuations and the effective size $\left(\alpha_{c}\right)$ of the attractive molecular interaction. Since the typical range of the dispersion forces in Lennard-Jones-like fluids is slighly greater than twice the equilibrium distance $\left(r_{e} \gtrsim \sigma\right)$ between two interacting particles of finite core size $\sigma$, we have reported on the upper axis of Figure 2 a rough estimate $\left[\ell_{q f}^{*} \approx \frac{1}{2}\right]$ of the limit where the correlation length will be comparable with the order of magnitude of the particle size. From the theoretical crossover fit, such a limit corresponds to $\mathcal{T}_{\bar{p}}^{*} \approx 1.3$ (here the supscript $\bar{p}$ is for a related particle property). Therefore, it is important to note that the effective crossover for the fluid subclass appears in the thermal field range $\mathcal{T}_{C O}^{*} \approx 0.5-1$ where $\ell_{q f}^{*} \lesssim 1$, as discussed below.

As illustrated by the curve labelled $C O$ in Figure $2 \mathrm{a}$, the rough estimate of the effective crossover around $\mathcal{T}_{C O}^{*} \approx 0.5-1$ corresponds to the following noticeable differences in $\gamma_{e f f}$-values obtained from fitting analyses [13, 35, 36, 37] of the isothermal compressibility data of xenon obtained from $p V T$ measurements [38, 39, 40]: for $\mathcal{T}^{*}<\mathcal{T}_{C O}^{*}, \gamma_{e f f}$-values are always greater than $\frac{\gamma_{I s i n g}+\gamma_{M F}}{2} \cong 1.12$ and increase to $\gamma_{I s i n g} \cong 1.24$ when $\mathcal{T}^{*} \stackrel{2}{\rightarrow} 0$; for $\mathcal{T}^{*} \geq \mathcal{T}_{C O}^{*}, \gamma_{\text {eff }}$-value is slightly constant and close to unity (see also the Figure 1 in reference [33|). This general trend is observed whatever the selected pure fluid, as already noted in references [13, 14]. In spite of the difficulty in determining the precise shape of the $\gamma_{e f f^{-}}$ variation in this crossover range where $\ell_{q f}^{*} \lesssim 1$, we may expect to not observe a collapse onto an unique crossover curve for different pure fluids (see also our analysis [29] of crossover behavior for the renormalized order parameter of several pure fluids in the non-homogeneous domain). As a matter of fact, the massive renormalization scheme is not appropriate when $\ell_{q f}^{*} \lesssim 1$. We have illustrated this situation by the hypothetic curves labelled $C O$ and 8 in Figure $2 \mathrm{~b}$ where a possible decrease of $\nu_{\text {eff }}$ to zero occurs crossing $\mathcal{T}_{C O}^{*}$. Such a zero-value [significatively different from the (mean-field) $\frac{1}{2}$-value] should be the result of an expected value $\ell_{q f}^{*}=$ const $\cong \frac{1}{2}$ ( limiting curve $m$, analogous to the one of Figure 1) related to a constant value of the direct correlation length (however, as already noted, $\ell_{q f}^{*} \approx \frac{1}{2}$ is not here a "master" value whatever the pure fluid). At large temperature distance to the critical point (i.e. for $\mathcal{T} \gg \mathcal{T}_{C O}$ ), such a limit means that the direct correlation between interacting particles at equilibrium position $\left(r_{e} \cong \frac{\alpha_{c}}{2}\right)$ inside the (short-ranged) critical interaction cell, can mainly contribute to the local density fluctuations. We recall that, along the critical isochore in the homogeneous phase, $\frac{1}{Z}$, i.e. the number of particle in the critical interaction cell, and $\alpha_{c}$, i.e. the size of the critical interaction cell, are two quantities which are sligthly dependent on the temperature range (since the critical isochore is closely a straight line in the $p ; T$ diagram). Such a conjectured microscopic situation is realistic and similar to the results obtained from molecular numerical simulation of a Lennard-Jones like fluid, precisely in the temperature range $T^{*}=\frac{k_{B} T}{\varepsilon_{L J}}>2$ (which corresponds to $\mathcal{T}^{*}>1.5$ for the monoatomic rare gases such as argon, krypton and xenon). In that situation, only the first peak of the static structure factor is observed to be significant in a reduced density $\left[\rho^{*}=\frac{\rho\left(\sigma_{L J}\right)^{d}}{m_{\bar{p}}} \approx 0.3\right]$ range including the reduced critical density $\rho_{c}^{*} \approx 0.3\left(\varepsilon_{L J}\right.$ and $\sigma_{L J}$ are the two characteristics parameters of the Lennard-Jones like potential). However, at such "low" density and "high" temperature ranges, the associated coordination number $(\lesssim 4)$ is too small to infer validity of a mean-field approximation of the attractive interaction.

\section{CONCLUSION}

We provide an asymptotic description for the correlation length singular behavior of the one-component fluid subclass. The master critical crossover behavior of this subclass can be observed up to $\mathcal{T}^{*} \approx 0.1$ (or $\ell_{q f}^{*} \approx 3$ ). The one-component fluid subclass then corresponds to the simplest situation in the $\Phi_{d=3}^{4}(n=1)$-model where the starting point for $u_{4}>0$ (in usual renormalized trajectories [32]), is certainly very close to the ideal RG trajectory between the Gaussian and the Wilson-Fisher fixed points [32]). Such a supplementary constraint, is not a necessity in the field theory framework [16]. In a complementary work, we will show that this constraint certainly takes origin in the description of the critical point vicinity by finite (linearized) thermodynamics, which provides 
complete understanding for the master thermodynamic properties normalized at the volume scale $\left(\alpha_{c}\right)^{d}$ of the critical interaction cell.
[1] H. L. Swinney and D. L. Henry, Phys. Rev. A 8, 2586 (1973).

[2] H. Güttinger and D. S. Cannell, Phys. Rev. A 24, 3188 (1981).

[3] M. Bonetti, P. Calmettes, and C. Bervillier, J. Chem. Phys. 119, 8542 (2003).

[4] J. S. Lin and P. W. Schmidt, Phys. Rev. Lett. 33, 1265 (1974); Phys. Rev. A 10, 2290 (1974).

[5] K. Ohbayashi and A. Ikushima, Phys. Lett. 46A, 131 (1973).

[6] F. Zhong, M. Barmatz, and I. Hahn, Phys. Rev. E 67, 021106 (2003).

[7] V. G. Puglielli and N. C. Ford, Jr., Phys. Rev. Lett. 25, 143 (1970).

[8] D. S. Cannell, Phys. Rev. A 12, 225 (1975).

[9] B. S. Maccabee and J. A. White, Phys. Rev. Lett. 27, 495 (1971).

[10] J. H. Lunacek and D. S. Cannell, Phys. Rev. Lett. 27, 841 (1971).

[11] D. Sullivan, PhD Thesis, University of Bristol, 2000, unpublished.

[12] M. Bonetti, G. Romet-Lemonne, P. Calmettes, and M.C. Bellissent-Funel, J. Chem. Phys. 112, 268 (2000).

[13] Y. Garrabos, Thesis, University of Paris VI, 1982, unpublished.

[14] Y. Garrabos, J. Phys. (France) 44, 281291 (1985) [for an english version see https://hal.ccsd.cnrs.fr/ccsd-00015956 (15 Dec. 2005) or http://fr.arxiv.org/abs/cond-mat/0512347; 45, 197-206 (1986).

[15] Y. Garrabos, Preprint 2005 [see https://hal.ccsd.cnrs.fr/ccsd-00015988 (16 Dec. 2005) or http://fr.arxiv.org/abs/cond-mat/0512408.

[16] C. Bagnuls and C. Bervillier, Phys. Rev. E 65, 066132 (2002).

[17] C. Bagnuls and C. Bervillier, J. Phys. (France) - Lettres 45, L-95 (1984).

[18] C. Bagnuls and C. Bervillier, Phys. Rev. B 32, 7209 (1985).

[19] C. Bagnuls, C. Bervillier, D. I. Meiron, and B.G. Nickel, Phys. Rev. B 35, 3585 (1987); 65, 149901(E) (2002).

[20] see for example J. Zinn-Justin, Euclidean Field Theory and Critical Phenomena, 3rd ed. (Oxford University Press, 1996).

[21] see for example M. A. Anisimov and J. V. Sengers, Critical Region, in "Equations of state for fluids and fluid mixtures". Part I, Ed. J. V. Sengers, R. F. Kayser, C. J. Peters, and H. J. White, Jr. (Elsevier, Amsterdam, The Netherlands, 2000) pp. 381-434.

[22] B. Widom, J. Chem. Phys. 43, 3898 (1965).

[23] R. Guida and J. Zinn-Justin, J. Phys. A: Math. Gen. 31, 8103 (1998).

[24] F. J. Wegner, Phys. Rev. B 5, 4529-4536 (1972).

[25] We admit that the molecular mass, $m_{\bar{p}}$, of each constitutive fluid particle is a known quantity, in order to infer $N$ value from total mass $\left(M=N m_{\bar{p}}\right)$ measurement of the amount of fluid matter filling the container of measured total volume $V$.

[26] see for example M. Modell and R. C. Reid, Thermodynamics and its Applications. 2nd ed. (Prentice Hall, New York, 1983).

[27] J. O. Hirschfelder, C. F. Curtiss, and R. B. Bird, Molecular Theory of Gases and Liquids (corrected edition), (Wiley, New York, 1964).

[28] A. Kostrowicka Wyczalkowska, J. V. Sengers, and M. A. Anisimov, Physica A 334, 482 (2004).

[29] Y. Garrabos, B. Le Neindre, R. Wunenburger, C. Lecoutre-Chabot, and D. Beysens, Int. J. Thermophys. 23, 997 (2002).

[30] K. G. Wilson, Phys. Rev. B 4, 3174 (1971).

[31] K. G. Wilson and J. Kogut, Phys. Rep. C 12, 75 (1974).

[32] see for example, C. Bagnuls and C. Bervillier, Cond. Matter Phys. 3, 559-575 (2000), and references therein.

[33] C. Bagnuls, C. Bervillier, and Y. Garrabos, J. Phys. (France)- Lettres 45, L-127 (1984).

[34] Y. Garrabos, F. Palencia, C. Lecoutre, and C. Bervillier, preprint (2005).

[35] W. T. Estler, R. Hocken, T. Charlton, and L. R. Wilcox, Phys. Rev. A 12, 2118 (1975); R. J. Hocken and M. R. Moldover, Phys. Rev. Lett. 37, 29 (1976).

[36] J. V. Sengers and M. R. Moldover, Phys. Lett. 66A, 44 (1978).

[37] J. M. H. Levelt-Sengers, W. L. Greer, and J. V. Sengers, J. Phys. Chem., 5, 1 (1976).

[38] H. W. Habgood and W. G. Schneider, Can. J. Chem. 32, 98 (1954).

[39] A. Michels, T. Wassenaar, and P. Louwerse, Physica 20, 99 (1954).

[40] J. A. Beattie, R. J. Barriault, and J. S. Brierley, J. Chem. Phys. 19, 1219 (1951); 19, 1222 (1951). 


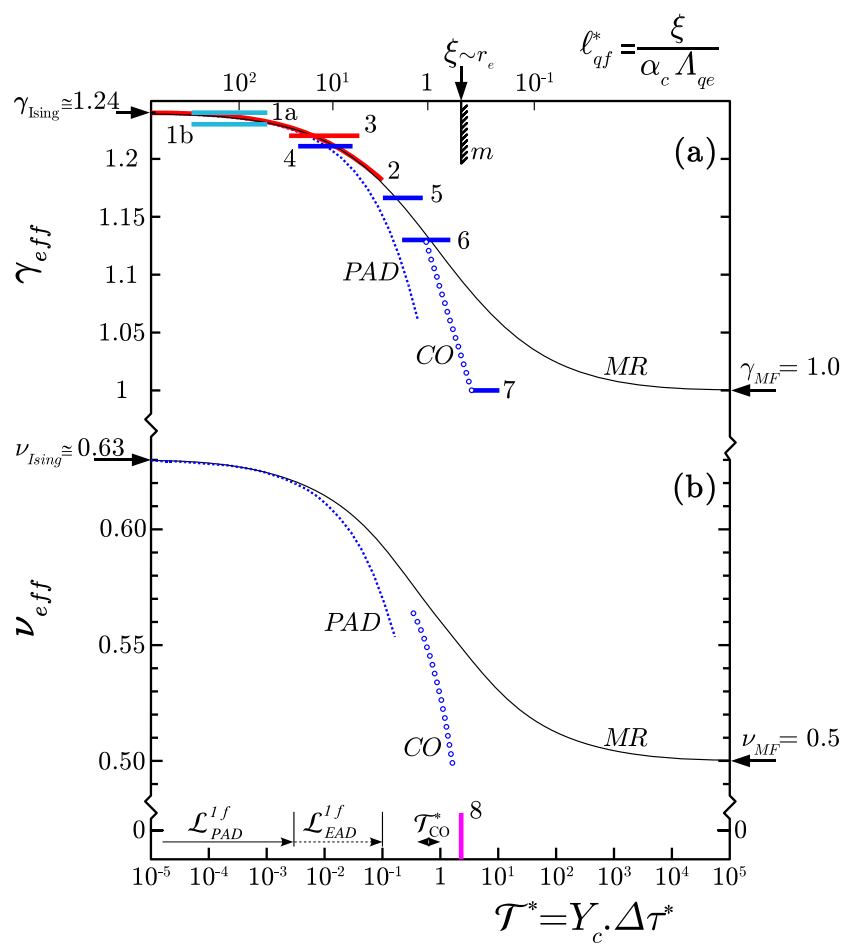

Figure 2: (Color on line) Variations of the effective exponents $\gamma_{\text {eff }}$ (part a) and $\nu_{\text {eff }}$ (part b) as a function of the renormalized thermal feld $\mathcal{T}^{*}$ (lower axis) or as a function of the renormalized correlation length $\ell_{q f}^{*}$ (upper axis). The full curves labelled $M R$ represent the $\gamma_{\text {eff }}$ and $\nu_{\text {eff }}$ theoretical variations obtained from the respective mean crossover functions (see Ref. 34] and text). The dashed curves labelled $P A D$ correspond to the exponent variation in the preasymptotic domain obtained from a Wegner expansion restricted to the first confluent correction [see equation (21) and text ], whose thermal field validity extends up to $\mathcal{L}_{P A D}^{1 f} \lesssim 310^{-3}$ (see the corresponding arrow in $\mathcal{T}^{*}$ axis). In the extended asymptotic domain (labelled $E A D$ ), the experimental master behavior is well-represented by the theoretical crossover function [see equation (31)], whose thermal field validity extends up to $\mathcal{L}_{E A D}^{1 f} \lesssim 0.1$ (see the corresponding arrow in $\mathcal{T}^{*}$ axis). The schematic curves labelled $C O$ and the curve 8 (part b) correspond to the hypothetized effective crossover for pure fluids in a thermal field range $0.5 \lesssim \mathcal{T}_{C O}^{*} \lesssim 1$, (see the corresponding double arrow in $\mathcal{T}^{*}$ axis). The 1 -to- 7 curves for $\gamma_{\text {eff }}$-variations refer [see also Ref. (33])] to experimental estimations of the isothermal compressibility for xenon: $1 a$ and 1b) from interferometric measurements (see Refs. [35] and [36], respectively); 2) from light scattering measurements (see Ref. [2]); 3) from turbidity and ligth scattering measurements (see Ref. [13]); 4-to-7) from $p V T$ measurement analysis (see Refs. 13, 14, 37]). For the selected $p V T$ measurements see Refs. 38, 39, 40]. For curve $m$ see the Figure 1 legend and text. 